\title{
Evaluation of Indoor Air treatment by two pilot-scale biofilters packed with compost and compost-based material
}

\author{
M. Ondarts, C. Hort, V. Platel \& S. Sochard \\ Université de Pau et des Pays de l'Adour, Laboratoire de Thermique, \\ Energétique et Procédés, Département Génie des Procédés, Equipe \\ "Traitement des effluents gazeux" Site de Tarbes, France
}

\begin{abstract}
The interest in Indoor Air Quality (IAQ) has increased this past decade. Indeed, many studies focused on Indoor Air analysis have pointed out many potential health risks and non-negligible associated costs. But only a few studies have dealt with the adaptation of industrial processes like sorption or photocatalysis and with development of new processes. Moreover, the use of these processes is still limited by the characteristics of this pollution: lots of components with different properties (Volatile Organic Compounds (VOC), aldehyde, inorganic compounds), competition phenomena between these pollutants, and low concentrations.

Biofiltration is currently used to treat high flow rate effluents with low concentrations and various pollutants, so this technology seems adapted for IA treatment. This study focuses on the evaluation of the performance of biofilters packed with compost and compost-based material (a mixture of compost/activated carbon). Indeed, compost is a natural material that possesses a lot of microorganisms, as well as good physical properties (water retention, $\mathrm{pH}$ ) and nutriment content. The model effluent is constituted of ten compounds (aldehyde, aromatic, chlorinated, inorganic...) at low concentrations (sub-ppmv), which have been chosen for their ubiquity in indoor environments, their different physical and chemical properties (solubility, vapor pressure, biodegradability) and their potential health risks in chronic exposures.

The pilot scale is constituted by a gas generator (permeation module) feed with high quality zero air and two biofilters with compost or compost/activated carbon (AC) based-bed material. VOCs are analysed by Gas Chromatography/Mass Spectrometry coupled with a cryogenic preconcentrator.

The first part of this study has demonstrated the possibility of generating a continuous sub-ppmv pollutants mixture. A simple analysis method that demonstrates this adaptation to this range of concentration is presented. Keywords: Indoor Air, VOCs, biofiltration, sub-ppmv level, model effluent.
\end{abstract}




\section{Introduction}

During the past decade, the interest in Indoor Air Quality (IAQ) has increased. Indeed, the progress in analytical chemistry methods and the multiplication of Indoor Air (IA) studies as RIOPA (Relationship between Indoor, Outdoor, Personal Exposure), NHEXAS (National Human Exposure Assessment Survey) in United States or Expolis in Europe have allowed an increase in knowledge of IA pollution. Indoor Air pollution presents two specificities. On one hand, IA pollution is constituted by a great number of various pollutants: few hundred compounds, including hydrocarbon compounds as alkanes, aromatic compounds, chlorinated compounds as trichloroethylene (TCE), pesticides, or inorganic compounds as ammonia, nitrogen dioxide, were detected in large studies as NHEXAS [1] or Expolis [1]. On the other hand, concentrations of these pollutants are generally very low and vary between $0.1 \mu \mathrm{g} \mathrm{m}^{-3}$ to $100 \mu \mathrm{g} \mathrm{m}^{-3}$ for the most concentrated [1-3]; these levels are higher than in outdoor: the ratio between indoor and outdoor pollutant concentrations is generally greater than 1 and can reach 20 or more [4]. There are lots of pollution sources: typically, building materials and use of cleaning products are the main sources [4]. Use of pesticide (acarina treatment, wood treatment), use of combustion apparatus and tobacco smoke are additional sources which can be specific from one country to another, and specific of practices of each country too [5].

Since the time spent in the indoors is close to $80 \%$, the chronic exposure to IA pollutants is the major source of exposure to pollution for human being. Moreover, different studies have observed or proposed relations between some indoor pollutants and some diseases: lung cancer could be linked to radon exposure, others cancers to pesticides, disorders of the reproduction to glycol ether and, at last, Sick Building Syndrome could be linked to multiple Volatile Organic Compounds (VOCs) [6]. Thus IAQ becomes a public health matter priority for public health organization as World Health Organization (WHO), United States Environmental Protection Agency (US-EPA) or governmental action as National Health Environment Plan in France [7].

Three ways can be investigated to remove IA pollution:

- $\quad$ suppression of pollutants sources: for example bio-materials, like tannin resin, are studied to replace formaldehyde in particle boards but this strategy cannot be applied to all buildings;

- $\quad$ increase the ventilation rate to dilute the pollution: this solution is not compatible with energy policy;

- use processes to remove pollutants.

Processes studied for IA treatment are current industrial processes that have to be adapted to IA pollution specificities. Indeed, concentrations are 1000 times lower than classical concentrations found in industrial effluents. Moreover, industrial treatments are adapted to specific chemical and physical properties, and so are limited for the treatment of various pollutants. Despite the present poor number of studies about IA treatment, many of classical processes have been studied in this aim. On one hand, a part of processes are based on the transfer of pollutants to another phase, which include adsorption [8], absorption 
[9], membrane process [10] and electrostatic separation [11]. Most of the studies and present commercial cleaners are based on adsorption process, most often on $\mathrm{AC}$ [8]. On the other hand, some processes are based on the degradation of pollutants, generally by oxidation, which include catalytic oxidation [12], UV photocatalysis [8], plasma and ionisation technologies [13], phytoremediation [14] and bioprocesses [15, 16].

All processes can present good efficiency, for example phytoremediation and plasma technology can respectively remove $75 \%$ and $50 \%$ of total VOCs (TVOC) $[14,13]$, UV photocatalysis can treat $83 \%$ of inlet nitrogen oxide [17].

However, many limitations were found during these processes studies. The first limitation, for treatments based on pollutant transfer is the necessity to regenerate the cleaning phase or to change it [8]. The fact that most of these processes are selective constitutes a second typical limitation. For example, adsorption on activated carbon is not adapted for compounds with low molecular weight as formaldehyde, which is a ubiquitous, and toxic compound in indoor. Membrane process presents by definition selectivity due to the choice of the membrane (hydrophobic or hydrophilic, pore size). The most observed others limitations are [8]:

- competition or inhibitory effect between different components;

- inhibition in presence of humidity;

- creation of by-products more dangerous or generating inhibition of the process.

The bioprocess has been studied for IA treatment since 1980 with Wolverton's work [16]. This work deals with potted plant biofilter for 3 compounds: formaldehyde, TCE, toluene. The efficiency of the process, which depends on the used plant, is closed to $80 \%$ for toluene. More recently, Darlington studied a trickling biofilter and observed great removal for aromatic compounds at low concentration [15].

In fact, biofilter process has properties that seem to be adapted to IA pollutants. First, lots of compounds present in indoor are successfully treated by biofiltration: efficiency closed to $100 \%$ can be obtained for biodegradable compounds as alcohol, acetate and efficiency from $40 \%$ to $100 \%$ can be obtained for more recalcitrant compounds like chlorinated compounds, aromatic or terpene [18]. Moreover, the biofiltration have been used for the treatment of waste air from publicly owned wastewater, which is effluent with low concentrations of pollutants [18]. This process is recommended for the treatment of large flow rate with low concentration [19].

Biofiltration is also a technology that is friendly for environment without chemical add products and which is relatively cheap. Thus biofiltration that seems to be an adapted technology has been chosen for IA pollutants removal. In order to evaluate this technology, the determination of the most representative experimental conditions is required: the composition of the model effluent, the concentrations of its pollutants and the flow rate have to be discussed. This paper describes the establishment of a pilot-scale, which allows one to obtain these conditions. 


\section{Protocol}

With the expansion of the IA cleaners market, especially for particular pollution, some organizations have established normalisations in order to test and qualify these cleaners. Different protocols can be differentiated according to the nature of the target pollution: particular pollution, biological pollution or chemical pollution. Attention is largely paid to particular pollution. Only a Japan protocol, JEM 1767, has been established and adopted as far as we know for chemical pollution [20]. It deals with odorous compounds linked to tobacco smokes. Two other protocols has been proposed but not adopted yet by the ASHRAE (American Society of Heating, Refrigerating and Air-Conditioning Engineers) [8] and the association EDF/CETIAT [20]. Table 1 presents the principal characteristics of these 3 protocols.

Table 1: $\quad$ Normalizated and non-normalizated protocol for chemical IA cleaners.

\begin{tabular}{|l|l|l|l|l|}
\hline Organisation & Date & $\begin{array}{l}\text { Configuration } \\
\text { test }\end{array}$ & $\begin{array}{l}\text { Target } \\
\text { Compounds }\end{array}$ & $\begin{array}{l}\text { Efficient } \\
\text { criterion }\end{array}$ \\
\hline $\begin{array}{l}\text { JEM 1467 } \\
\text { Japan }\end{array}$ & $\begin{array}{l}\text { March } \\
1995\end{array}$ & chamber & $\begin{array}{l}\text { ammonia } \\
\text { acethaldehyde } \\
\text { acetique acid }\end{array}$ & Lifespan \\
\hline $\begin{array}{l}\text { ASHRAE } \\
\text { USA }\end{array}$ & 2005 & chamber & 16 VOCs & CADR $^{\text {a }}$ \\
\hline $\begin{array}{l}\text { EDF/CETIAT } \\
\text { France }\end{array}$ & May 2006 & open system & toluene & CADR \\
\hline
\end{tabular}

a :Clean Air Delivery Rate

Three main differences can be noted in these 3 methods: efficient criterion, configuration of the test chamber and model pollutants. Two different efficient capacity criteria are used. Japan's protocol uses a criteria based on the lifespan of the cleaner calculated with a series of tests made until cleaner saturation is reached. The lifespan is expressed as a function of the number of cigarettes whose smoke can be treated. The 2 others protocols use the "Clean Air Delivery Rate" (CADR), product of flow rate (Q) and efficiency (RE) of the cleaner. The last one seems to be more adapted to different compounds.

The choice of model pollutants is a critical point for a good qualification of the cleaner. Indeed, all processes can have a good efficiency with compounds that present a good affinity for the process. However, one of the difficulties encountered in IA treatment is the presence of lots of chemical families with various properties.

This point is clearly proposed by ASHRAE's protocol: 16 compounds with different properties (alkanes, TCE, aromatic) are taken as model components. 
Usually, only few compounds are chosen in studies dealing with IA treatment; a low number of studies use more than three compounds as model pollutants.

We have chosen to take 10 compounds into account in our model effluent. The compounds usually chosen are formaldehyde and toluene as aromatic compound. Indeed, these compounds are ubiquitous in the indoors and their toxicity is clearly established. These two points constituted the first part of our choice of model compounds. Moreover, the various chemical and physical properties of the 10 pollutants have been taken into account to evaluate the capacity of biofiltration to treat a broad range of pollutants. This latest point is necessary to answer to the typical problem of IA pollution: multi-exposure.

Public health organizations have already established lists of priority compounds in indoor based on ubiquity and toxicity of pollutants. These lists are in agreement with criteria retained for the constitution of the model effluent we will generate. Indeed, three organizations lists were retained to establish the model effluent: WHO's [21] US-EPA's lists [22] (reference organizations for public health matter); and OIAQ list (Observatory of Indoor Air Quality) in order to take into account the national specificities of indoor air pollution [23].

The IA compounds properties can be very different from a component to another: the Henry coefficient of limonene is $10^{6}$ times higher than this of nitrogen dioxide, the biodegradability of 1-butanol is very good whereas TCE is known to be a xenobiotic compound [19].

Moreover, compounds concentrations are very different. Final concentrations are not obviously defined. Actually, only a few normalizations define good IA quality characteristics; only radon, for chemical pollution, is concerned by normalization. Table 2 presents some selected compounds median indoor concentrations in a French study and some toxicological reference concentrations recommended by WHO, US-EPA, ATSDR (Agency for Toxic Substances and Disease Registry) and Canadian directive.

Table 2: Medium IA concentration in France [23] and toxicological concentrations.

\begin{tabular}{|l|c|l|}
\hline $\begin{array}{l}\text { Priority Compound } \\
\text { in Indoor }\end{array}$ & $\begin{array}{c}\text { Concentrations } \\
\mathrm{mg} \mathrm{m}^{-3}\end{array}$ & \multicolumn{1}{|c|}{$\begin{array}{c}\text { Toxicological Ref. } \\
\mathrm{mg} \mathrm{m}^{-3}\end{array}$} \\
\hline Formaldehyde & $2.40 .10^{-2}$ & $\begin{array}{l}1.0 .10^{-2} \text { ATSDR } \\
1.0 .10^{-1} \text { Canada }\end{array}$ \\
\hline Toluene & $1.56 .10^{-2}$ & $\begin{array}{l}4.0 .10^{-1} \text { EPA } \\
3.0 .10^{-1} \text { ASTDR }\end{array}$ \\
\hline $\mathrm{TCE}^{\mathrm{NO}}{ }_{2}$ & $8.60 .10^{-4}$ & $2.0 .10^{-2}$ ASTDR \\
\hline Dichlorvos & $4.30 .10^{-2}$ & $\begin{array}{l}4.0 .10^{-2} \text { OMS } \\
1.0 .10-2 \text { Canada }\end{array}$ \\
\hline
\end{tabular}

a: Chronic exposition 
All the concentrations of the different compounds do not behave in the same manner with regard to toxicological threshold: some medium concentrations are lower than toxicological concentrations and some medium concentrations are higher than recommendations. However, only the Canadian directive includes the multi-exposure indoors. In this directive, the recommended value for formaldehyde depends on the presence and on the concentration of acetaldehyde and acroleine. Any other toxicological concentration is defined for multiexposure. Owing to this fact, and as it's recommended by NIOSH (National Institute for Occupational Safety and Health) for carcinogenic compounds, the treatment have to decrease the indoor pollutant concentrations as low as possible.

The last point discussed in indoor cleaner protocol is the configuration of the test unit. Chamber test is currently used and has been retained in JEM protocol and by the ASHRAE. However, the results can be strongly influenced by the sample point in the chamber. Moreover, for low pollutant concentrations, high sample volume is required and so can disturb the system. Open system with continuous feed seems to be more adapted and simplify the analytic part.

\section{Installation of pilot bench scale}

\subsection{Design of the biofilter}

Despite the recent progress in the comprehension of phenomena involved during biofiltration and so despite recent development of new biofilter models, the design of biofilter is still often empirical since these phenomena and then these models are very complex. "Empty Bed Time Retention" (EBRT) is usually used to design biofilter. Recommended EBRT varies between $20 \mathrm{~s}$ and $60 \mathrm{~s}$ respectively for low and high pollutant concentration [19]. Moreover, Darlington observed that elimination capacity of his bioprocess for indoor air treatment increases with the increase of the flow rate. The mass loading is then increased and tends towards classical mass loading in biofiltration. So, biofilter is designed for an EBRT of $20 \mathrm{~s}$. The second parameter is the depth of the bed. The recommended depth varies between 0.5 and $2 \mathrm{~m}$ [24] to minimize the pressure drop and the compaction of the bed. For the pilot bench scale, depth of the bed is $0.5 \mathrm{~m}$. The flow rate adapted to this depth, to the diameter of the column, and to the EBRT is $101 \mathrm{mn}^{-1}$.

The pilot bench scale can be divided into 3 parts:

1. generation of model effluent;

2. biofilter;

3. analytical apparatus.

\subsection{Effluent generation}

The concentration of each component varies between $30 \mu \mathrm{g} \mathrm{m}^{-3}$ to $100 \mu \mathrm{g} \mathrm{m}^{-3}$. This effluent is produced by a calibration gas generator (PUL200, Calibrage society, France) constituted with 2 permeation ovens with capacity of 5 components by oven. The range of concentration generated by the gas generator, 
with accuracy about $5 \%$ (according to the pollutant), is close to hundreds $\mu \mathrm{g} \mathrm{m}^{-3}$. The generator is fed by zero air with maximal concentration in carbonate compounds about $50 \mathrm{ppbv}$. In order to decrease the effluent concentration, a system enabling the dilution of the effluent (ratio 1:6) on one hand and the split of the gas generator outlet (ratio 1:10) on the other hand has been implemented. All regulations are performed with Brook Instrument flow meter with an accuracy of $1 \%$ of the flow range.

\subsection{Biofilter design}

Two biofilter supports are tested for this application: compost and mixture of compost and activated carbon (AC). Compost is a natural support known for its good degradation capacity: it presents natural microorganisms and nutriments content [19]. Moreover, compost is relatively cheap and allows waste valorisation. The mixture compost/AC has already been studied for its good removal capacity and its bulking agent property [19].

The two Biofilters are two columns of $100 \mathrm{~mm}$ inner diameter in borosilicate glass, which is a material with low adsorption capacity. Some distribution Teflon meshes are arranged at different levels of the biofilter in order to avoid gas preferential paths. A bubbler unit enables water saturation of the effluent. All pipes are in Teflon (pfa), which is inert.

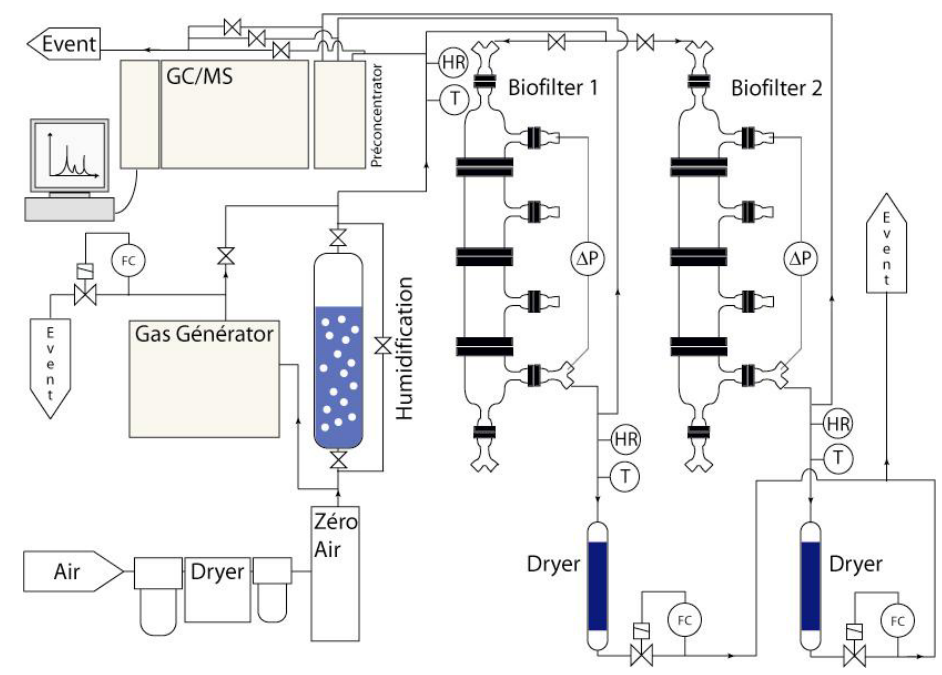

Figure 1: $\quad$ Schematic diagram of biofiltration unit.

\subsection{Analytical apparatus}

VOCs are analysed by Gas Chromatography/Mass Spectrometry (GC/MS, Trace MS Plus, Thermoelectron SA). First a cryogenic preconcentration removes water and carbon dioxide, which are responsible for interference in the analysis, and 
concentrates the effluent at the same time. Preconcentration is necessary because the MS sensibility is too low. The temperature program applied was $35^{\circ} \mathrm{C}$ during $5 \mathrm{mn}, 8^{\circ} \mathrm{C}$ until $100^{\circ} \mathrm{C}$ and $15^{\circ} \mathrm{C}$ until $200^{\circ} \mathrm{C}$. The detection mode applies is Single Ion Monitoring (SIM).

Figure 2 shows analytical result for a mixture of toluene, acetate butyl, limonene, undecane, TCE.

The nitrogen dioxide is analysed by an apparatus based on chemiluminescence with a detection limit about the ppbv level.

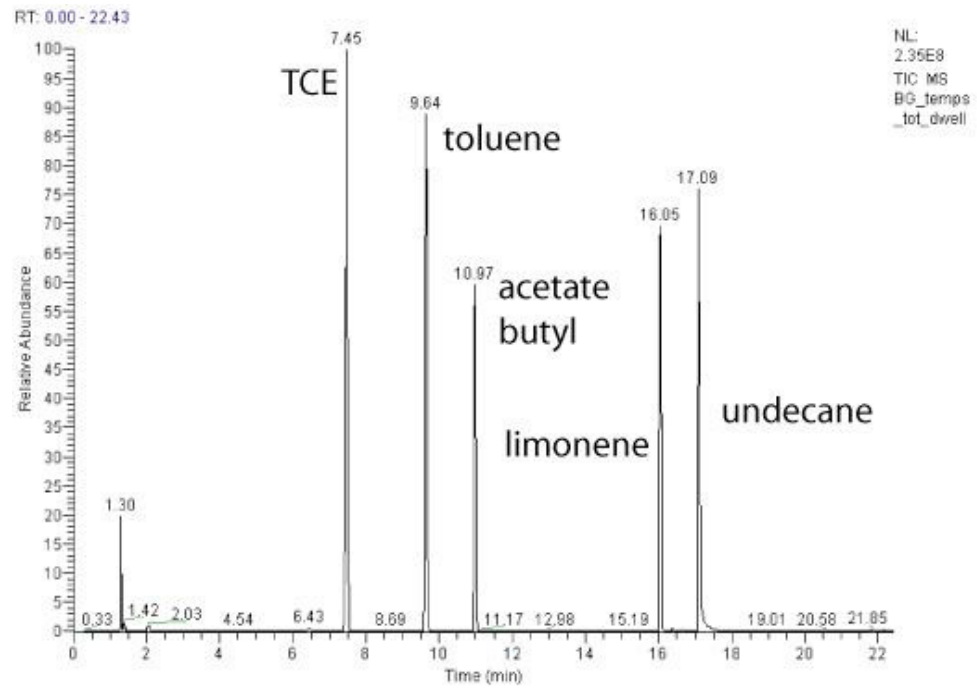

Figure 2: $\quad$ Typical chromatograph of effluent with concentration of $100 \mu \mathrm{g}$ $\mathrm{m}^{-3}$ for each compound.

\section{Conclusion}

The focus on IAQ, which started in the last decade, has increased in recent years. On one hand, public health organizations are more and more concerned with IAQ. On the other hand, lots of institutions as governments or industries focus on IAQ. Indeed, the poor Indoor Air Quality has been pointed out in numerous European studies and the implementation of treatment solutions seems to be unavoidable. Note that space industry is also concerned since the IA of cabin has to be treated. Moreover, the same problem holds for car industry. At the present time, treatment processes are not enough efficient: processes are always limited by inhibition, selectivity or byproducts problems. Moreover, the present knowledge is limited by tests with poor number of pollutants with high concentration with regard to real IA constitution.

This work has proposed a protocol based on the constitution of a representative effluent: the pollutants were selected for their occurrence in indoor, their toxicity and their different chemical and physical properties. The concentrations of pollutants are relatively close to IA concentrations. These 
different aspects have needed original technical solutions, which have been described. The generation of effluent with low concentration has been done. The analysis apparatus has been optimized and is efficient for sub-ppmv analysis.

The biofiltration technology seems to be adapted to IA treatment. Biofiltration is currently recommended for treatment of large effluent flow rate with low concentration of pollutants as shown in some past studies. The biofilter has been designed in agreement with literature and seems to be adapted.

\section{Acknowledgements}

This work was supported by ADEME (Environment and Energy Control Agency, France) and Hautes Pyrénées General Council, France.

\section{References}

[1] L. Mosqueron and V. Nedellec. Revues des enquêtes sur la qualité de l'air intérieur dans les logements en europe et aux etats-unis. Ethnical report, Observatoire de la Qualité de l'Air Intérieur, 2004.

[2] C.P. Weisel, J. Zhang, B.J. Turpin, M.T. Morandi, S. Colome, T.H. Stock, and D.M. Spektor. Relationships of indoor, outdoor and personal air (riopa), part i. collection methods and descriptive analyses. Technical report, Health Effects Institute, 2005.

[3] K. Saarela, T. Tirkkonen, J. Laine-Ylijoki, J.Jurvelin, M.J. Nieuwenhuijsen, and M. Jantunen. Exposure of population and microenvironmental distributions of volatile organic compound concentrations in the expolis study. Atmospheric Environment, 37: 5563-5575, 2003.

[4] R.D. Edwards, J. Jurvelin, K. Koistinen, K. Saarela, and M. Jantunen. Voc source identification from personal and residential indoor, outdoor and workplace microenvironment samples in expolis-Helsinki, Finland. Atmospheric Environment, 35: 4829-4841, 2001.

[5] M.S. Zuraimi, C.-A. Roulet, K.W. Tham, S.C. Sekhar, K.W.D. Cheong, N.H. Wong, and K.H. Lee. A comparative study of VOCs in Singapore and European office buildings. Building and Environment, 41: 316-329, 2006.

[6] A.P. Jones. Indoor air quality and health. Atmospheric Environment, 33: 4535-4564, 1999.

[7] AFSSE. Rapport de la commission d'orientation du plan national santé environnement. Technical report, Agence Française de Sécurité Sanitaire Environnementale, 2004.

[8] W. Chen, J.S. Zhang, and Z. Zhang. Performance of air cleaners for removing multiple volatile organic compounds in indoor air. ASHRAE Transactions, 111: 1101-1114, 2005.

[9] P.-C. Luo, Z.-B. Zhang, Z. Jiao, and Z.-X. Wang. Investigation in the design of a co2 cleaner system by using aqueous solutions of monoethanolamine and diethanolamine. Ind. Eng. Chem. Res., 42: 48614866, 2003. 
[10] S. Aguado, A.C. Polo, M.P. Bernal, J. Coronas, and J. antamaria. Removal of pollutants from indoor air using zeolite membranes. Journal of Membrane Science, 240: 159-166, 2004.

[11] T. Ito, N. Namiki, M. Lee, H. Emi, and Y. Otani. Electrostatic separation of volatile organic compounds by ionization. Environmental Sciences and Technology, 36: 4170-4174, 2002.

[12] Y. Sekine. Oxidative decomposition of formaldehyde by metal oxides at room temperature. Atmospheric Environment, 36: 5543-5547, 2002.

[13] S.L. Daniels. "On the ionization of air for removal of noxious effluvia"(air ionization of indoor environments for the control of volatile and particule contaminants with nonthermal plasmas generated by dielectric-barrier discharge. IEEE Transaction on plasma science, 4: 1471-1481, 30.

[14] R. A. Wood, M. D. Burchett, R. Alquezar, R. L. Orwell, J. Tarran, and F. Torpy. The potted-plant microcosm substantially reduces indoor air voc pollution: I. office field-study. Water, Air, and Soil Pollution, 175: 163$180,2006$.

[15] A.B. Darlington, J.F. Dat, and M.A. Dixon. The biofiltration of indoor air: air flux and temperature influences the removal of toluene, ethylbenzene and xylene. Environmental Sciences and technology, 35: 240-246, 2001.

[16] B.C. Wolverton and A. Johnson. Interior landscape plants for indoor air pollution abatement. Technical report, National Aeronautics and Space Administration, 1989.

[17] C.H. Ao and S.C. Lee. Indoor air purification by photocatalyst tio2 immobilized on an activated carbon filter installed in an air cleaner. Chemical Engineering Science, 60: 103-109, 2005.

[18] R. Iranpour, H.H.J. Cox, M.A. Deshusses, and E.D. Schroeder. Literature review of air pollution control biofilters and biotrickling filters for odor and volatile organic compound removal. Environmental Progress, 24: 254-267, 2005.

[19] J.S. Devinny, M.A. Deshusses, and T.S. Webster. Biofiltration for air pollution control. Lewis Publishers, 1999.

[20] P. Blondeau, A. Ginestet, Dr F. Sqinazi, Pr F. de Blay, M. Ott, B. Ribot, and D. Frochot. Mise en place de protocoles de qualification des appareils d'épuration d'air, rapport final. Technical report, CETIAT, EDF RD, Mairie de Paris, Université de la Rochelle, Les Hôpitaux Universitaires de Strasbourg, 2006.

[21] WHO. Air Quality Guidelines - second Edition. WHO Regional Publications, 2000.

[22] P.K. Johnston, G. Hadwen, J. McCarthy, and J.R. Girman. A screeninglevel ranking of toxic chemicals at levels typically found in indoor air. In Indoor Air, 2002.

[23] L. Mosqueron and V. Nedellec. Hiérarchisation sanitaire des paramètres mesurés dans les bâtiments par l'observatoire de la qualité de l'air intérieur. Technical report, Observatoire de la Qualité de l'Air Intérieur, 2002.

[24] I. Datta and D.G. Allen. Biotechnology for odor and air pollution control. Springer, 2005. 